\title{
Materials and Solid Mechanics
}

\author{
Md Sadiqul Hasan Talukder \\ B.Sc. in Mechanical Engineer Department of Mechanical Engineering Rajshahi University of Engineering \& \\ Technology, Bangladesh.
}

\begin{abstract}
This research work deals with the implication of modern retailing at not only in Dhaka, Bangladesh but also the whole district in Bangladesh with main objectives to find out technological activity, impact on modern welfare..
\end{abstract}

Keywords: Stress, Strain, Elasticity, Linear momentum, Moh'r Circle

\section{Introduction}

The application of the principles of mechanics to bulk matter is divided into the machanics of fluids and the mechanics of solids. The entire subject is often called continuum mechanics, particularly when we adopt the useful model of matter as being continuously divisible, making no reference to its discrete structure at microscope length. Solid mechanics is concerned with the stressing, deformation and failure of solid materials and structures. What, then, is a solid? Any material, fluid or solid, can support normal forces. Water at the base of a pond, air in an automobile tire, the stones of a Roman arch, rocks at base of a mountain, the skin of a pressurized airplane cabin, a stretched rubber band and the bones of a runner all support force in that way (some only when the force is compressive. We call a material solid rather than fluid if it can also support a substantial shearing force over the time scale of some natural process or technological application of interest.

\section{Headings}

Here also concepts of stress, strain and elasticity are important. Robert Hooke discovered it in 1660 but published in 1678. French mathematicians in the early 1800's of a theory of small transverse displacements and vibrations of elastic plates. This theory was developed in preliminary from by Sophie Germain and partly improved upon by Simeon Denis Poisson in the early 1810's; they considered a flat plate as an elastic plane which resists curvature. Navier gave a definitive development of the correct energy expression and governing differential equation a few years later.

\section{Indentations And Equations}

Concepts of stress, strain and elastic The English scientist Robert Hooke discovered in 1660, but published only in 1678, the observation that for many materials that displacement under a load was proportional to force, thus establishing the notion of (linear) elasticity but not yet in a way that was expressible in terms of stress and strain. E. Mariotte in France published similar discoveries in 1680 and, also, reached an understanding of how beams like those studied by Galileo resisted transverse loadings or, more precisely, resist the torques caused by those transverse loadings, by developing extensional and compressional deformations, respectively, in material fibers along their upper and lower portions. It was for Swiss mathematician and mechanician James Bernoulli (1654-1705) to observe, in the final paper of his life, in 1705, that the proper way of describing deformation was to give force per unit area, or stress, as a function of the elongation per unit length, or strain, of a material fiber under tension. Swiss mathematician and mechanician Leonhard Euler (17071783), who was taught mathematics by James' brother John Bernoulli (1667-1748), proposed, among many contributions, a linear relation between stress ${ }_{-}$and strain in $_{1} 1727$, of form ${ }_{-}=\mathrm{E}_{-}$where the coefficient $\mathrm{E}$ is now generally called Young's modulus after English naturalist Thomas Young who developed a related idea in 1807.

Beams, columns, plates, shells The 1700's and early 1800's were a productive period in which the mechanics of simple elastic structural elements were developed well before the beginnings in the 1820's of the general three-dimensional theory. The development of beam theory by Euler, who generally modeled beams as elastic lines which resist bending, and by several members of the Bernoulli family and by Coulomb, remains among the most immediately useful aspects of solid mechanics, in part for its simplicity and in part because of the pervasiveness of beams and columns in structural technology. James Bernoulli proposed in his final paper of 1705 that the curvature of a beam was proportional to bending moment. Euler in 1744 and John's son, Daniel Bernoulli (1700-1782) in 1751 used the theory to address the transverse vibrations of beams, and Euler gave in 1757 his famous analysis of the buckling of an initially straight beam subjected to a compressive loading; the beam is then commonly called a column. Following a suggestion of Daniel Bernoulli in 1742, Euler in 1744 
introduced the strain energy per unit length for a beam, proportional to the square of its curvature, and regarded the total strain energy as the quantity analogous to the potential energy of a discrete mechanical system.

Elasticity, general theory Linear elasticity as a general three-dimensional theory was in hand in the early 1820's based on Cauchy's work. Simultaneously, Navier had developed an elasticity theory based on a simple corpuscular, or particle, model of matter in which particles interacted with their neighbors by a centralforce attractions between particle pairs. As was gradually realized following works by Navier, Cauchy and Poisson in the 1820's and 1830's, the particle model is too simple and makes predictions concerning relations among elastic moduli which are not met by experiment. In the isotropic case it predicts that there is only one elastic constant and that the Poisson ratio has the universal value of 1/4. Most subsequent development of the subject was in terms of the continuum theory. Controversies concerning the maximum possible number of independent elastic moduli in the most general anisotropic solid were settled by English mathematician George Green in 1837, through pointing out that the existence of an elastic strain energy required that of the 36 elastic constants, relating the six stress components to the six strains, at most 21 could be independent. Scottish physicist Lord Kelvin (William Thomson) put this consideration on sounder ground in 1855 as part of his development of macroscopic thermodynamics, in much the form as it is known today, showing that a strain energy function must exist for reversible isothermal or adiabatic (isentropic) response, and working out such results as the (very modest) temperature changes associated with isentropic elastic deformation.

Stress concentrations and fracture In 1898 G. Kirsch derived the solution for the stress distribution around a circular hole in a much larger plate under remotely uniform tensile stress. The same solution can be adapted to the tunnel-like cylindrical cavity of circular section in a bulk solid. His solution showed a significant concentration of stress at the boundary, by a factor of three when the remote stress was uniaxial tension. Then in 1907 the Russian mathematician

G. Kolosov, and independently in 1914 the British engineer Charles Inglis, derived the analogous solution for stresses around an elliptical hole. Their solution showed that the concentration of stress could become far greater as the radius of curvature at an end of the hole becomes small compared to the overall length of the hole. These results provided the insight to sensitize engineers to the possibility of dangerous stress concentrations at, for example, sharp re-entrant corners, notches, cut-outs, keyways, screw threads, and the like in structures for which the nominal stresses were at otherwise safe levels. Such stress concentration sites are places from which a crack can nucleate.

In addressing any problem in continuum or solid mechanics, we need to bring together the following considerations: (1) The Newtonian equations of motion, in the more general form recognized in the subsequent century by Euler, expressing conservation of linear and angular momentum for finite bodies (rather than just for point particles), and the related concept of stress as formalized by Cauchy; (2) Consideration of the geometry of deformation and thus expression of strains in terms of gradients in the displacement field; and (3) Use of relations between stress and strain that are characteristic of the material in question, and of the stress level, temperature and time scale of the problem considered.

These three considerations suffice for most problems in solid and structural mechanics for simple materials. They must be supplemented for solids undergoing diffusion processes in which one material constituent moves relative to another (as of interest sometimes for a fluid-infiltrated soils or petroleum reservoir rocks), and in cases for which the induction of a temperature field by deformation processes and the related heat transfer cannot be neglected. The latter cases require that we supplement the above three considerations with the following:

(4) Equations for conservation of mass of diffusing constituents; (5) The first law of thermodynamics, which introduces the concept of heat flux and relates changes in energy to work and heat supply, and (6) Relations which express the diffusive fluxes and heat flow in terms of spatial gradients of appropriate chemical potentials and of temperature. Also, in many important technological devices, electric and magnetic fields affect the stressing, deformation and motion of matter. Examples are provided by piezoelectric crystals and other ceramics for electric or magnetic actuators, and the coils and supporting structures of powerful electromagnets. In these cases, we must add the following: (7) Scottish physicist James Clerk Maxwell's set of equations which interrelate electric and magnetic fields to polarization and magnetization of material media, and to the density and motion of electric charge; and (8) Augmented relations between stress and strain which now, for example, express all of stress, polarization and magnetization in terms of strain, electric field and magnetic intensity, and of temperature. Also, the second law of thermodynamics, combined with the principles mentioned above, serves to constrain physically allowed relations between stress, strain and temperature in (3). Such considerations were first brought to bear in a purely mechanical context by Green in 1837, as based on the existence of a strain energy which generalized, for a continuum, the potential energy of the discrete dynamical systems of analytical mechanics; they were later rooted in the development of macroscopic thermodynamics by Kelvin. The second law also constrains the other types of relations described in (6) and (8) above. Such relations are commonly referred to as constitutive relations. 
Linear and Angular Momentum Principles: Stress, and Equations of Motion Let x denote the position vector of a point in space as measured relative to the origin of a Newtonian reference frame; $x$ has the components $\left(\mathrm{x}_{1}, \mathrm{x}_{2}, \mathrm{x}_{3}\right)$ relative to a Cartesian set of axes, fixed in the reference frame, which we denote as the 1 , 2 and 3 axes, Figure 1. (This form of notation proves more convenient for the subject than a convention which may be more familiar to many readers, in which positions are denoted as $(\mathrm{x}, \mathrm{y}, \mathrm{z})$ and the reference axes as the $\mathrm{X}, \mathrm{Y}$ and $\mathrm{Z}$ axes.) Suppose that a material occupies the part of space considered and let $\mathrm{v}=\mathrm{v}(\mathrm{x}, \mathrm{t})$ be the velocity vector of the material point which occupies position $\mathrm{x}$ at time $\mathrm{t}$; that same material point will be at position $\mathrm{x}+\mathrm{v} d t$ an infinitesimal time interval dt later. Let $\_=\_(x, t)$ be the mass density of the material. Here $\mathrm{v}$ and _ are macroscopic variables. What we idealize in the continuum model as a material point, moving as a smooth function of time, will correspond on molecular (or larger but still "microscopic") length scales to a region with strong fluctuations of density and velocity. In terms of phenomena at such scales, _ corresponds to an average of mass per unit of volume, and _ $v$ to an average of linear momentum per unit volume, as taken over spatial and temporal scales that are large compared to those of the microscale processes but still small compared to those of the intended application or phenomenon under study. Thus $\mathrm{v}$ of the continuum theory is a massweighted average velocity, from the microscopic viewpoint. (There do not generally exist tractable formulations of macroscopic mechanics when such a separation of scales does not apply. This is an important area of research since many important phenomena involving the fracture of solids and fine scale inelastic deformation processes do not have a clear separation of length scales. Large scale digital computer simulations of systems of discrete particles allow some of the simpler of such cases to be addressed; these include atomistic modeling of fracture and plastic flow processes in small regions of crystals, and flows of granular solids with highly idealized particle interactions.)

Fig: 1. Coordinate system; position (x) and velocity (v) vectors; body force $\mathrm{f} d \mathrm{~V}$ acting on element $\mathrm{dV}$ of volume, and surface force $\mathrm{T} d \mathrm{~S}$ acting on element dS of surface.

\section{FIG: 1}

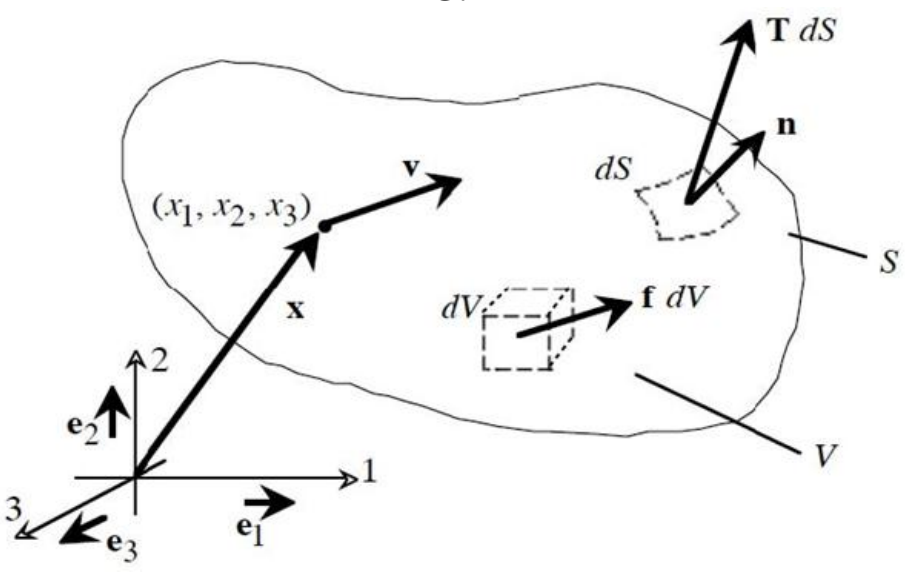

We observe that an infinitesimal element of material occupying volume $\mathrm{dV}$ at $\mathrm{x}$ moves and distorts in such a way that _ $\mathrm{dV}$, which corresponds to the (conserved) mass of the element, remains constant. The linear momentum of the element is $\_\mathrm{V} \mathrm{dV}$ and its angular momentum relative to the coordinate origin is given as the vector, or cross, product $\mathrm{x}_{-}\left(\overline{(}_{-} \mathrm{v} \mathrm{dV}\right)$. Thus the linear momentum $\mathrm{P}$, and angular momentum $\mathbf{H}$ relative to the coordinate origin, of the matter instantaneously occupying any volume $\mathrm{V}$ of space are then given by summing up the linear and angular momentum vectors of each element of material. Such summation over infinitesimal elements is represented mathematically by the integrals

$\mathbf{P}={ }_{-} \mathbf{v ~ d V}, \mathbf{H}=_{-} \mathbf{x}_{-} \mathbf{v} \mathrm{dV}$

V_V

We limit attention to situations in which relativistic effects can be ignored.

Let $\mathbf{F}$ denote the total force and $\mathbf{M}$ the total torque or moment (relative to the coordinate origin) acting instantaneously on the material occupying any arbitrary volume V. The basic laws of Newtonian mechanics are the linear and angular momentum principles that

$\mathbf{F}=\mathrm{d} \mathbf{P} / \mathrm{dt}, \mathbf{M}=\mathrm{d} \mathbf{H} / \mathrm{dt}$,

where time derivatives of $\mathbf{P}$ and $\mathbf{H}$ are calculated following the motion of the matter which occupies $\mathrm{V}$ at time $t$. Newton's focus was on matter in situations for which the particle point of view is valid, so that only $\mathbf{F}=\mathrm{dP} / \mathrm{dt}$ is 
required. It was Euler who recognized the need for the two vectorial laws of motion for general finite bodies; he explicitly stated the pair of laws $\mathbf{F}=\mathrm{d} \mathbf{P} / \mathrm{dt}$ and $\mathbf{M}=\mathrm{d} \mathbf{H} / \mathrm{dt}$ in 1776, then for a rigid body, but had implicitly recognized them as early as 1752 . When either $\mathbf{F}$ or $\mathbf{M}$ vanish, these equations of motion correspond to conservation of linear or angular momentum. An important, very common, and non-trivial class of problems in solid mechanics involves determining the deformed and stressed configuration of solids or structures that are in static equilibrium; in that case the relevant basic equations are $\mathbf{F}=\mathbf{0}$ and $\mathbf{M}=\mathbf{0}$. The understanding of such conditions for equilibrium, at least in a rudimentary form, long predates Newton. Indeed, Archimedes of Syracuse (3rd Century BC), the great Greek mathematician and arguably the first theoretically and experimentally minded physical scientist, understood these equations at least in a nonvectorial form appropriate for systems of parallel forces. That is shown by his treatment of the hydrostatic equilibrium of a partially submerged body and his establishment of the principle of the lever (torques about the fulcrum sum to zero) and the concept of center of gravity. Archimedes' approach to natural philosophy is now the standard model of science but was overshadowed for about 2000 years by Aristotle's (4th Century BC) style of ex-cathedra, if sometimes insightful, speculation. The Dutch mathematician and engineer, Simon Stevin recognized the vectorial nature of the equations $\mathbf{F}=\mathbf{0}$ and $\mathbf{M}=\mathbf{0}$ for static equilibrium, developing the parallelogram law of vectorial force addition in 1586 and correctly analyzing the principle of the lever for systems of nonparallel forces.

Stress vector and equations of motion in integral form We now assume that $\mathbf{F}$ and $\mathbf{M}$ derive from two type of forces, namely body forces $\mathbf{f}$, like gravitational attractions, defined such that force $\mathbf{f} \mathrm{dV}$ acts on volume element dV (see Figure 1), and surface forces which represent the mechanical effect of matter immediately adjoining that along the surface, $\mathrm{S}$, of the volume $\mathrm{V}$ that we consider. Cauchy formalized in 1822 a basic assumption of continuum mechanics that such surface forces could be represented as a vector distribution $\mathbf{T}$, defined so that $\mathbf{T}^{\text {force }} \mathrm{dS}$ is an element of force acting over the area dS of the surface, Figure 1. (Shortly, we will want to define a stress vector $\mathbf{T}$, of which this $\mathbf{T}$ will be a part; it is typically the dominant part for solid materials.) Thus, for any arbitrarily chosen region, like in Figure 1, we assume that total force and torque acting can be written, respectively, as

${ }_{T}$ force $_{\mathrm{dS} \mathbf{x}_{-} \mathrm{T}}$ force $\mathrm{dS}$

$\mathbf{F}=+\mathbf{f} \mathrm{dV}$, and $\mathbf{M}=+\mathbf{x}_{-} \mathbf{f} \mathrm{dV}$.

S_V_S_V

These should now be equated, respectively, to the rates of change of linear and angular momentum, $\mathrm{dP} / \mathrm{dt}$ and $\mathrm{d} \mathbf{H} / \mathrm{dt}$.

To calculate $\mathrm{d} \mathbf{P} / \mathrm{dt}$, note that the integrand for $\mathbf{P}$ contains the product _dV times $\mathbf{v}$. Since the mass element _dV is invariant in the motion, it has zero time derivative, and we need only calculate the derivative of its velocity $\mathbf{v}$, which is acceleration $\mathbf{a}$. However, the correct expression for $\mathrm{d} \mathbf{P} / \mathrm{dt}$ contains the term which has just been motivated plus a second term:

${ }_{T}$ mom. flux $\mathrm{dS}_{\mathrm{dS}}$

$\mathrm{dP} / \mathrm{dt}=_{-} \mathbf{a d V}+$

-V_S

That second term arises because there is a microscopic motion, in general, relative to the massmom.flux

averaged macroscopic motion, and that relative motion causes some momentum flux $\mathbf{T}$ per unit area, across the surfaces $S$. In the continuum model, the surface $S$ moves through space such that the velocity of the surface in a direction normal to itself is $\mathbf{n}_{-} \mathbf{v}$, where $\mathbf{n}$ is the unit outer normal to $S$ at the point considered and $\mathbf{v}$ is the velocity at that point. Since $\mathbf{v}$ is a mass-weighted average of fluctuating velocities on a molecular (or larger microscopic) scale, this assures that there is no mass transferred across $\mathrm{S}$, but not that there is no momentum transferred; $\mathbf{T}$ accounts for that latter transfer. In a similar way, the rate of change of angular momentum can be calculated and we obtain the expression

$\mathbf{x}_{-}{ }_{\mathrm{T}} \mathrm{mom}$. flux $\mathrm{x}_{\mathrm{dS}}$

$\mathrm{d} \mathbf{H} / \mathrm{dt}=\mathbf{x}_{-} \mathbf{a} \mathrm{dV}+$.

-V_S

The acceleration $\mathbf{a}=\mathbf{a}(\mathbf{x}, \mathrm{t})=\mathrm{d} \mathbf{v} / \mathrm{dt}$ is calculated such that the time derivative of $\mathbf{v}$ is taken following the motion of a material point. Thus $\mathbf{a}(\mathbf{x}, \mathrm{t}) \mathrm{dt}$ corresponds to the difference between $\mathbf{v}(\mathbf{x}+\mathbf{v} \mathrm{dt}, \mathrm{t}+\mathrm{dt})$ and $\mathbf{v}(\mathbf{x}, \mathrm{t})$. Also, in deriving the expression for $\mathrm{d} \mathbf{H} / \mathrm{dt}, \mathbf{v}=\mathrm{d} \mathbf{x} / \mathrm{dt}$ has been used, with the derivative again following the motion, and it has been noted that $\mathbf{v}_{-} \mathbf{v}=\mathbf{0}$.

We now define the stress, or traction, vector $\mathbf{T}$ by 


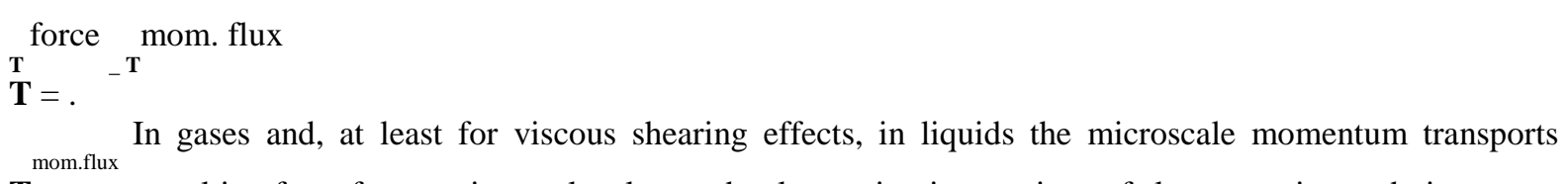

$\mathbf{T}$, resulting from fast moving molecules randomly moving into regions of slower motion and vice-versa, are the main contribution to $\mathbf{T}$. In solids they generally are a small contribution, especially at low temperatures compared to that for melting. It will generally be the case that microscopic mass elements moving at a velocity different from $\mathbf{v}$, and hence contributing to $\mathbf{T}^{\text {mom.flux }}$, will undergo collisional interactions with other mass elements within a short distance of the surface element considered, reducing their speed, on average, to v. This delivers impulsive forces $-\mathbf{T}$ per unit area in the near vicinity of the surface so, including their effect, it is legitimate to refer to the total stress vector $\mathbf{T}$ as a force per unit area, as is often done in the literature of the subject.

Then , using the definition of $\mathbf{T}$, when we equate the expressions for $\mathbf{F}$ and $\mathbf{M}$ above to those for $\mathrm{dP} / \mathrm{dt}$ and $\mathrm{d} \mathbf{H} / \mathrm{dt}$, we obtain the equations of motion, in integral form, for a continuum,

$\mathbf{T d S}+\mathbf{f} \mathrm{dV}=_{-} \mathbf{a} \mathrm{dV}, \mathbf{x}_{-} \mathbf{T d S}+\mathbf{x}_{-} \mathbf{f d V}=_{-} \mathbf{x}_{-} \mathbf{a} \mathrm{dV}$

${ }_{-} \mathrm{S}_{-} \mathrm{V}{ }_{-} \mathrm{V}_{-} \mathrm{S} \mathrm{V}_{-} \mathrm{V}$ V

We now assume these to hold good for every conceivable choice of region $\mathrm{V}$.

Stress components. Nine quantities ${ }_{-}(\mathrm{ij}, \mathrm{j}=1,2,3)$ called stress components may be defined at each point of the medium; these will vary with position and time, ${ }_{-{ }_{\mathrm{ij}}}={ }_{-{ }_{\mathrm{ij}}}(\mathbf{x}, \mathrm{t})$, and have the following interpretation. Suppose that we consider an element of surface dS through a point $\mathbf{x}$ with dS oriented so that its outer normal (pointing away from the region $\mathrm{V}$, bounded by

$S)$ points in the positive $x_{i}$ direction, where $i$ is any of 1,2 or 3 . Then $-_{-i 1},{ }_{-i 2}$ and ${ }_{-i 3}$ at $\mathbf{x}$ are defined as the Cartesian components of the stress vector $\mathbf{T}$ (call it $\mathbf{T}^{(\mathrm{i})}$ ) acting on this dS. Figure

2 shows the components of such stress vectors for faces in each of the three coordinate directions. To use a vector notation with $\mathbf{e}_{1}, \mathbf{e}_{2}$ and $\mathbf{e}_{3}$ denoting unit vectors along the coordinate axes (Figure 1), (i) $={ }_{-i 1} \mathbf{e}_{1}+{ }_{-i 2} \mathbf{e}_{2}+{ }_{-i 3} \mathbf{e}_{3}$.

Thus the stress $-_{-\mathrm{ij}}$ at $\mathbf{x}$ is the stress in the $\mathrm{j}$ direction associated with an i-oriented face through point $\mathbf{x}$; the physical dimension of the $-_{\mathrm{ij}}$ is [Force]/[Length] $]^{2}$. The components $-_{11},{ }_{-22}$ and $-_{33}$ are stresses directed perpendicular, or normal, to the faces on which they act and are normal stresses; the ${ }_{-_{\mathrm{ij}}}$ with $\mathrm{i}_{-} \mathrm{j}$ are directed parallel to the plane on which they act and are shear

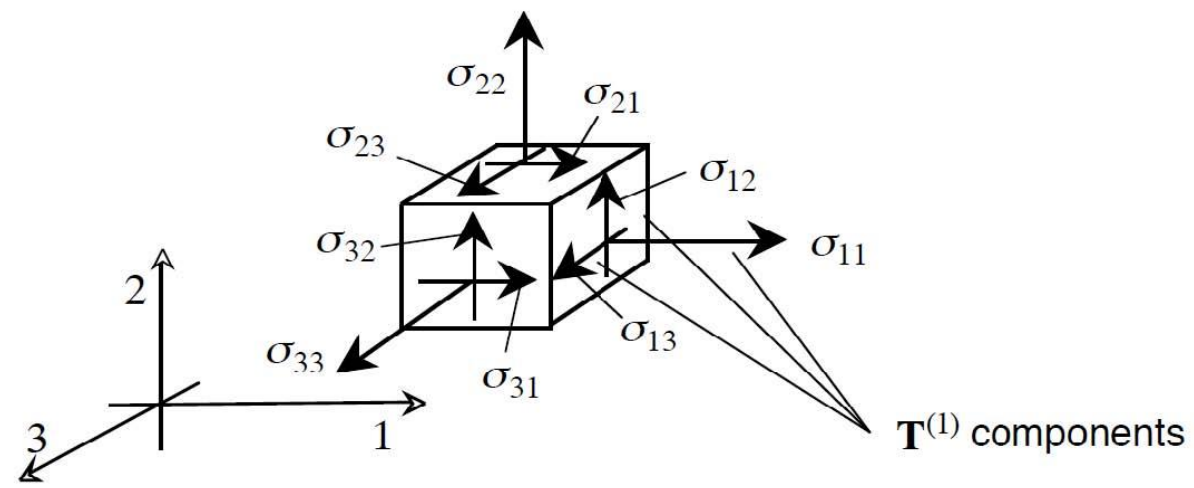

FIG: 2

FIG 2. Stress components; first index denotes plane, second denotes direction.

By hypothesis, the linear momentum principle applies for any volume V. If we first apply it to a small region including a general position $\mathbf{x}$, and consider the limit of the resulting equation as both the volume $\mathrm{V}$ and bounding surface area $S$ of the region approach zero, so that the region shrinks onto point $\mathbf{x}$. We can observe that the volume integrals, when divided by area $S$, approach zero in that limit. Thus, for such choices of region, the linear momentum principle requires that we set to zero the limit of $(1 / S) \mathbf{T d S}$ as $S$ approaches zero. Consider a thin sliver of material at $\mathbf{x}$, 
_S(Figure 3) with thin direction along the $\mathrm{x} 1$ axis, let that thickness approach zero, and then let the diameter of the region approach zero so that it shrinks onto $\mathbf{x}$. We thus derive that $\mathbf{T}^{(-1)}+\mathbf{T}^{(1)}=\mathbf{0}$, which is a special case of the action-reaction principle. (It, like other variants of the action-reaction principle, can be regarded as a derivable consequence of the hypothesis that the linear momentum principle applies for every choice of region, including two subregions which act upon one another and exert the forces referred to as the action and reaction.) The result tells us that, for any direction $\mathrm{i}$,

$\mathbf{T}^{(-\mathrm{i})}=-\mathbf{T}^{(\mathrm{i})}=-{ }_{-\mathrm{i} 1} \mathbf{e}_{1}-{ }_{-\mathrm{i} 2} \mathbf{e}_{2}-{ }_{-\mathrm{i} 3} \mathbf{e}_{3}$

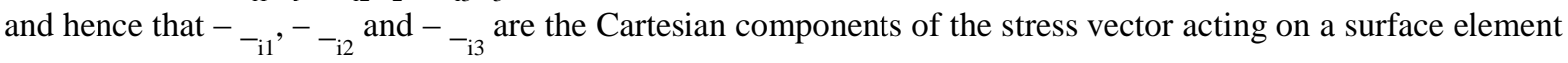
$\mathrm{dS}$ through $\mathbf{x}$ whose outer normal points in the negative i direction.

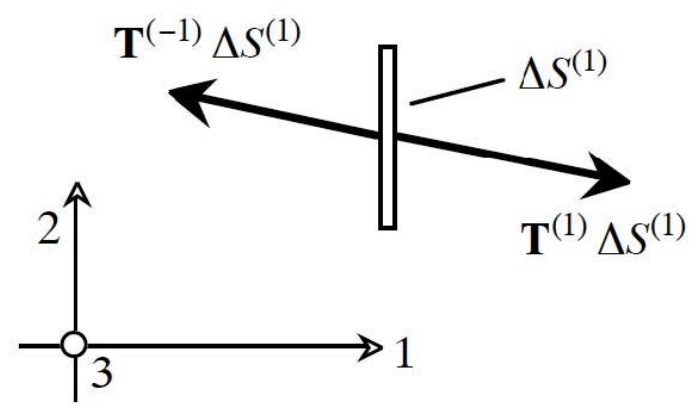

FIG: 3

FIG 3. Linear momentum principle leads to action-reaction, $\mathbf{T}^{(-1)}+\mathbf{T}^{(1)}=\mathbf{0}$.

Next we consider a small tetrahedron (Figure 4) at $\mathbf{x}$ with inclined face having outward unit normal vector $\mathbf{n}$, and other three faces oriented perpendicular to the three coordinate axes. Let $\mathbf{S}$ denote the area of the inclined face and $\mathrm{S}^{(1)}, \mathrm{S}^{(2)}$ and $\mathrm{S}^{(3)}$ the areas of the faces with outer normals respectively in the negative 1,2 and 3 directions; we note from geometry that $\mathrm{S}^{(\mathrm{i})} / \mathrm{S}=\mathrm{ni}$ (this also leads to the correct result when the normal to face $S^{(i)}$ points in the positive i direction, in which case $n i<0$ ). Letting the size of the tetrahedron approach zero, so that it shrinks onto $\mathbf{x}$, the linear momentum principle requires that $\mathbf{T}+\mathrm{n} 1 \mathbf{T}^{(-1)}+\mathrm{n} 2 \mathbf{T}^{(-2)}+\mathrm{n} 3 \mathbf{T}^{(-3)}=\mathbf{0}$. Thus, using the expression above for the $\mathbf{T}^{(-\mathrm{i})}$ we derive the result that the stress vector $\mathbf{T}$ on a surface

element through $\mathbf{x}$ with outward normal $\mathbf{n}$ can be expressed as a linear function of the ${ }_{-\mathrm{ij}}$ at $\mathbf{x}$. The relation is such that the $\mathbf{j}$ component of the stress vector $\mathbf{T}$ is

$3 \mathrm{~T}_{\mathrm{j}-} \mathbf{e}_{\mathrm{j}-} \mathbf{T}=\mathrm{n}_{1-1 \mathrm{j}}+\mathrm{n}_{2-2 \mathrm{j}}+\mathrm{n}_{3-3 \mathrm{j}}={ }_{-} \mathrm{n}_{\mathrm{i}-\mathrm{ij}}(\mathrm{j}=1,2,3) \mathrm{i}=1$

Stress transformation in a plane, Mohr circle Stress transformation in a plane is often of interest. Referring to Figure 7, suppose that the in-plane stress components acting in the $\mathrm{x}_{1}, \mathrm{x}_{2}$

plane are given, as in the upper left of the figure. We wish to find the in-plane stress components acting across a surface which is tilted about the $\mathrm{x} 3$ axis at angle ${ }_{-}$, measured positive anti-

clockwise relative to the $\mathrm{x} 1$ axis as shown. That surface has unit normal $\mathbf{n}$ and we let $\mathbf{s}$ be an

orthogonal unit vector along the surface in the $\mathrm{x}_{1}, \mathrm{x}_{2}$ plane, also as shown. The normal stress to be determined is called $_{-{ }_{n}}$ and the shear stress ${ }_{-n s}$; see the upper center diagram in Figure 7.

Letting $\mathbf{T}$ be the stress vector on the inclined plane, we obtain the stress components as ${ }_{{ }_{n}}=\mathbf{n}_{-} \mathbf{T}$ and ${ }_{{ }_{n}}=\mathbf{s}_{-}$ $\mathbf{T}$. Thus, recalling the previous expression for $\mathbf{T}$,

33333333

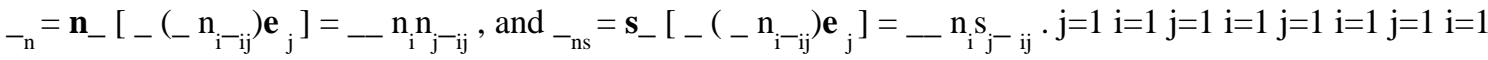

Since $\mathbf{n}=\left(\sin _{-}, \cos _{-}, 0\right)$ and $\mathbf{s}=\left(\cos _{-}, \sin _{-}, 0\right)$, these reduce (recalling that $\left.{ }_{-12}=_{-}{ }_{21}\right)$ to

$2^{-} 11^{+}{ }^{-} 22_{2}^{-} 22-11$

$-_{\mathrm{n}}={ }_{-11} \sin -_{-12} \sin \cos _{-}+_{-22} \cos { }_{-}=+\cos 2_{--_{12}} \sin 2_{-}$,

22

${ }^{-} 22-11$

$-_{\text {ns }}=-_{11} \sin _{-} \cos +_{-}+_{-12}\left(\cos ^{2}-\sin _{-}^{2}\right)+_{-22} \sin { }_{-} \cos -=\sin 2_{-}+_{-12} \cos 2_{-}$. 
2 A little analysis shows that the latter expressions are the parametric equations (with parameter _ ) of a circle in a Mohr plane whose axes are ${ }_{-n}$ and ${ }_{-}$; ; the circle is called the Mohr circle. It

$\sqrt{\left(\sigma_{22}-\sigma_{11}\right)^{2} / 4+\sigma_{122}^{2}}$

has center at $\left(-_{11}+{ }_{-22}\right) / 2$ along the ${ }_{-\mathrm{n}}$ axis, has radius

$(-22-11) / 4+_{-12}$, and rotation

of the inclined interface anti-clockwise by _ in the physical $\left(\mathrm{x}_{1}, \mathrm{x}_{2}\right)$ plane corresponds to anti-clockwise rotation by $2 \_$in the Mohr plane.
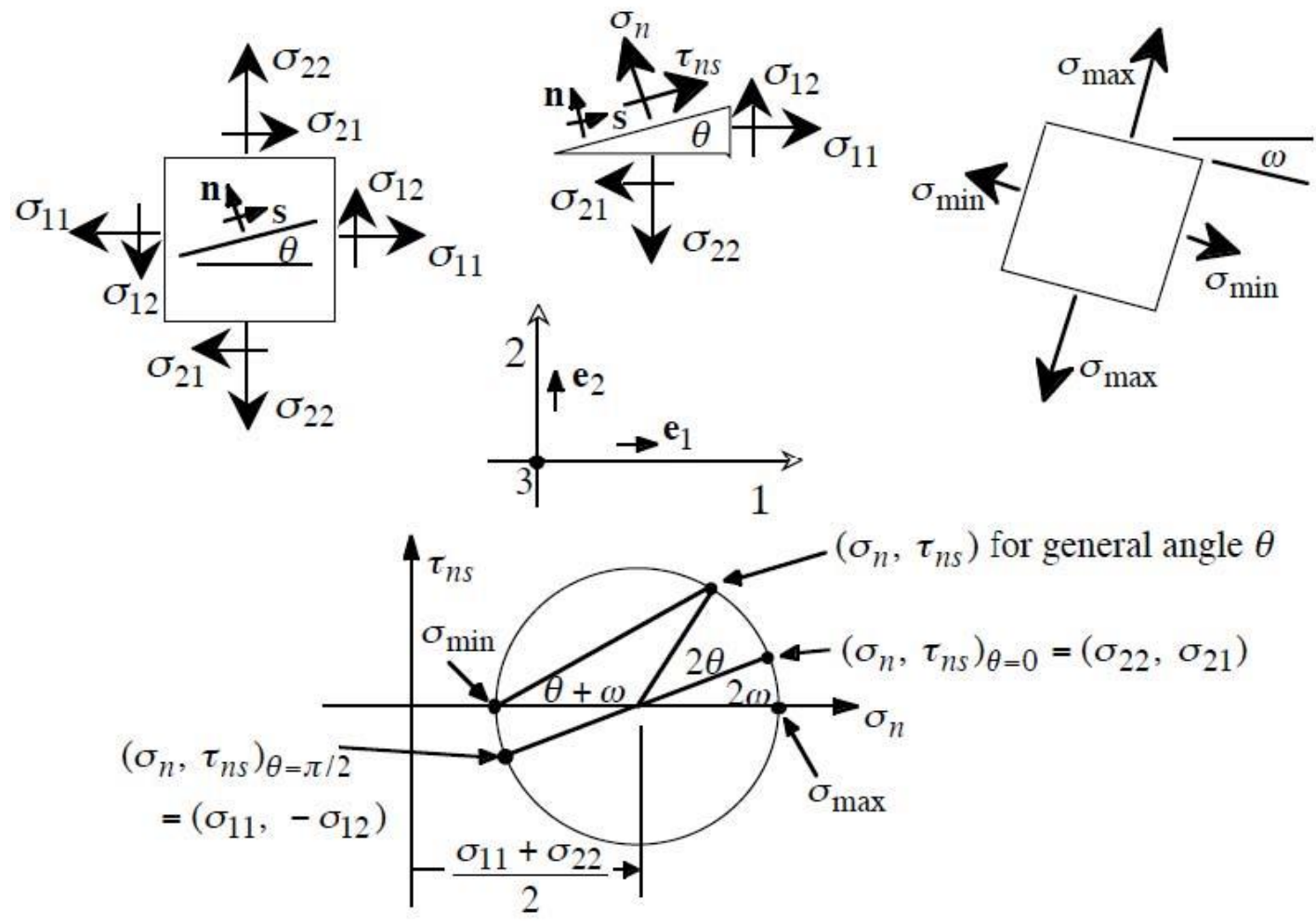

FIG: 4

FIG 4. Moh'r circle representation of stress transformation in a plane. A general stress state is shown at the upper left. The circle is used to determine the normal stress ${ }_{-n}$ and shear stress ${ }_{n}$ s , upper center, acting on a plane inclined at angle _.

The quickest way to construct the Mohr circle is usually to identify two points which lie on it, such uniquely locating the circle given that its center lies on the ${ }_{-n}$ axis. Thus, we first observe that $\left({ }_{n},{ }_{-}\right)=\left({ }_{22}\right.$, $\left.{ }_{-21}\right)$ must be the point on the circle corresponding to ${ }_{-}=0$, and then that $\left({ }_{-},{ }_{-}{ }_{n s}\right)=\left({ }_{-11},-_{12}\right)$ must be another point on it, corresponding to ${ }_{-}=\ldots$.

Both of those points are labeled in Figure 7. Once the circle is drawn, the stress state for a general orientation at angle ${ }_{-}$is given by rotating in the same sense around the circle, by angle $2_{-}$, from the point $\left({ }_{22},{ }_{21}\right)$ on it corresponding to $\_=0$. As seen in the figure, an angle $2_{-}$

may be defined as that marked, and then ${ }_{-}{ }_{\ldots}$ is the face orientation for which the maximum in-plane normal stress ${ }_{-\max }$ acts. That orientation is shown in the upper right of the figure, where ${ }_{\text {max }}$ and the least in-plane normal stress ${ }_{\text {min }_{\text {min }}}$ are the extremity points of the Mohr circle along the ${ }_{-n}$ axis. Finally, by an elementary geometric relation, the angle ${ }_{-}{ }_{-}$between the general orientation considered and that of the maximum in-plane normal stress can be identified as marked. 
Professional Experiences

Incase of Reaction it is described as belows:

At Aristopharma:

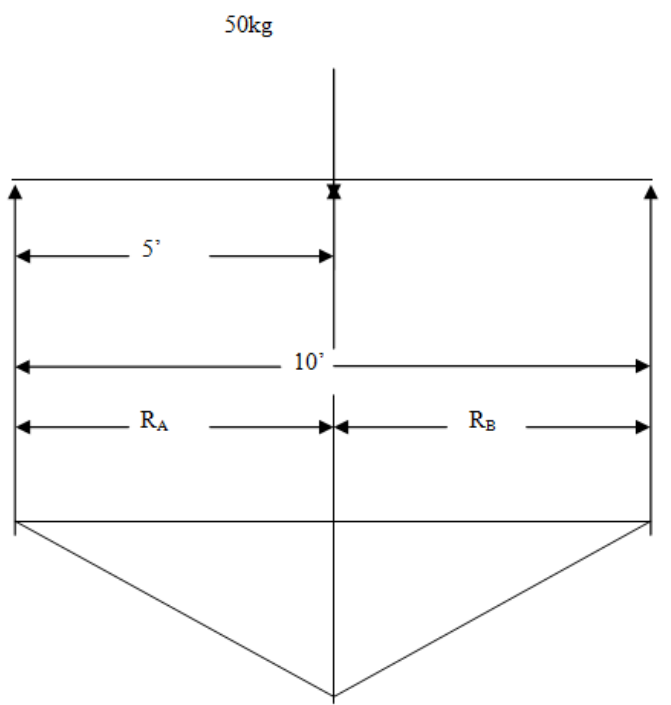

Here,

$\mathrm{SM}_{\mathrm{A}}=0$

Or, $50 \times 5-R_{B} \times 10=0$

Or, $\mathrm{R}_{\mathrm{B}}=(250 / 10) \mathrm{kg}=25 \mathrm{~kg}=\mathrm{R}_{\mathrm{A}}$

From, Diagram it has been shown that $\mathrm{R}_{\mathrm{A}=} \mathrm{R}_{\mathrm{B}}$

At Radiant:

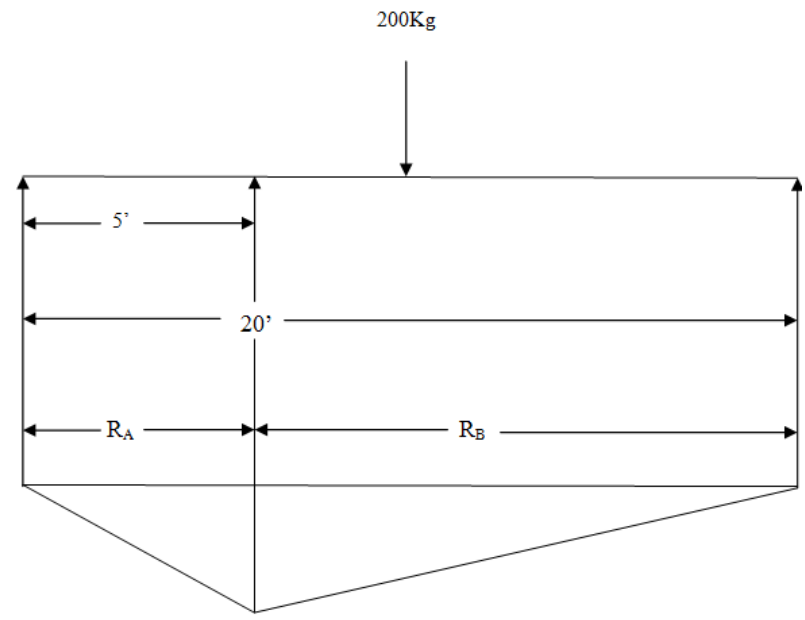

Here,

$\mathrm{SM}_{\mathrm{A}}=0$

Or, $200 \times 5-R_{B} \times 20=0$

Or, $\mathrm{R}_{\mathrm{B}}=(1000 / 20) \mathrm{kg}=500 \mathrm{~kg}$

Therefore, $R_{\mathrm{A}} / \mathrm{R}_{\mathrm{b}=} 200 / 500=2 / 5$

From, Diagram it has been shown that $\mathrm{R}_{\mathrm{A}=} 2 / 5 \mathrm{R}_{\mathrm{B}}$

\section{Limitation:}

\section{Conclusion}

1. Shear stress and modulus must be appropiate.

2. Sometimes to find out Reaction of the beam is very difficult.

Though it has limitations but modern era is very dependable on these. Specially in pharmaceutical sector these are very effective. Hence, all kinds safety for human is possible by this system. So, this system is absolutely welcome for modern era. 